\title{
ABSOLUTE AND UNCONDITIONAL CONVERGENCE
}

\author{
M. S. MACPHAIL
}

A series $\sum_{n=1}^{\infty} x_{n}$ whose terms are elements of a Banach space $B$ is said to converge absolutely if $\sum\left|x_{n}\right|$ converges, unconditionally if every rearrangement converges. If a series converges absolutely it converges unconditionally; absolute convergence and unconditional convergence are equivalent in any finite-dimensional Euclidean space, whereas in $L^{2}$, for instance, there are simple examples of series which converge unconditionally but not absolutely. The question in what spaces the two definitions are equivalent is left open by Banach [ 1 , p. 240]. ${ }^{1}$ It may be conjectured that unconditional convergence does not imply absolute convergence in any infinite-dimensional space (cf. $[2$, p. 30]), but the problem does not seem to have been explicitly treated. The purpose of this note is to obtain a criterion for the equivalence of the two notions in a given Banach space. As examples of its use we shall show that unconditional convergence does not imply absolute convergence in the spaces $L$ and $l$. The result for $L$ is already known (see for example [3, p. 45]), but the result for $l$ has not, to the author's knowledge, been stated elsewhere.

We shall need the following definitions. Let $S$ be any (finite) sequence of elements $\xi_{1}, \xi_{2}, \cdots, \xi_{p}$ in $B$. We do not require that the $\xi_{i}$ be all distinct, and we shall understand by addition, $S_{1}+S_{2}$, the mere adjoining of the two sequences. Thus we might write $(1,2,2,3)+(3,2,1)=(1,2,2,3,3,2,1)$. By $c S$ ( $c$ real $)$ we understand the sequence $c \xi_{1}, c \xi_{2}, \cdots, c \xi_{p}$. We shall use two norms,

$$
|S|=\sum\left|\xi_{i}\right|, \quad|S|^{*}=\sup _{\sigma}\left|\sum_{\sigma} \xi_{i}\right|,
$$

where $\sigma$ is any subset of $1,2, \cdots, p$. It is easily verified that $\left|S_{1}+S_{2}\right|=\left|S_{1}\right|+\left|S_{2}\right|,\left|S_{1}+S_{2}\right|^{*} \leqq\left|S_{1}\right|^{*}+\left|S_{2}\right|^{*},|c S|=|c||S|$, and $|c S|^{*}=|c||S|^{*}$. Define further, when $|S| \neq 0$,

$$
G(S)=|S| * /|S| \text {. }
$$

Note that $0<G(S) \leqq 1$. Let $g=\inf G(S)$, taken over all sequences $S \subset B$.

TheOREm. ${ }^{2}$ If $g=0$, unconditional convergence does not imply absolute convergence in $B$. If $g>0$, the two are equivalent.

Received by the editors July 25, 1946.

${ }^{1}$ Numbers in brackets refer to the references cited at the end of the paper.

2 Suggested to the author by Garrett Birkhoff, in conversation. 
Proof. (i) If $g=0$, we can find a sequence $\left\{S_{n}\right\}$ such that $G\left(S_{n}\right)<4^{-n}$. Put $c_{n}=2^{n} /\left|S_{n}\right|$, and $S_{n}^{\prime}=c_{n} S_{n}$. Then $\left|S_{n}^{\prime}\right|=c_{n}\left|S_{n}\right|=2^{n}$, and $\left|S_{n}^{\prime}\right|^{*}<4^{-n}\left|S_{n}^{\prime}\right|=2^{-n}$. A well known necessary and sufficient condition for unconditional convergence of a series $\sum x_{n}$ is that given $\epsilon>0$, we can find $N$ such that $\left|\sum_{8} x_{n}\right|<\epsilon$, where $s$ is any finite set of integers, all greater than $N$. From this it is easily seen that the series formed by summing the elements of $S_{1}^{\prime}+S_{2}^{\prime}+S_{3}^{\prime}+\cdots$ converges unconditionally but not absolutely.

(ii) If $g>0$, we may write $|S| \leqq|S|^{*} / g$, and it is now easily seen that unconditional convergence implies absolute convergence, and so the two are equivalent.

COROLLARY 1. Unconditional convergence does not imply absolute convergence in $L$.

PRoof. Let $r_{n}(t)$ denote the Rademacher orthogonal functions on $0 \leqq t \leqq 1$, defined by $r_{n}(t)=2 e_{n}(t)-1$, where $e_{n}(t)$ is the $n$th digit in the binary expansion of $t$, the value 0 being assigned at points that have two such expansions. Thus $r_{n}(t)$ takes the values -1 and +1 alternately on intervals of length $1 / 2^{n}$.

We shall show that if $S_{n}=\left(r_{1}, r_{2}, \cdots, r_{n}\right)$, we have

$$
\left|S_{n}\right|^{*} /\left|S_{n}\right| \rightarrow 0 \text {, }
$$

from which the desired result follows by the theorem. Evidently $\left|S_{n}\right|=n$. Moreover, when $\sigma$ is a subset containing $p$ of the numbers $1,2, \cdots, n$, use of the Schwarz inequality and the orthogonality of the functions $r_{k}(t)$ gives

$$
\int_{0}^{1}\left|\sum_{\sigma} r_{k}(t)\right| d t \leqq\left\{\int_{0}^{1}\left|\sum_{\sigma} r_{k}(t)\right|^{2} d t\right\}^{1 / 2}=p^{1 / 2}
$$

Hence, since $p \leqq n,\left|S_{n}\right|^{*} \leqq n^{1 / 2}$. Therefore (1) holds, and the proof is complete.

COROLlaRy 2. Unconditional convergence does not imply absolute convergence in $l$.

Proof. Since there is no necessity for the sets $S_{n}$ to have common elements, we may write

$$
\begin{aligned}
& S_{1}=\{(-1, \quad 1, \quad 0, \quad 0,0, \cdots)\}, \\
& S_{2}=\{(-1,-1, \quad 1, \quad 1, \quad 0, \quad 0, \quad 0, \cdots) \text {, } \\
& (-1, \quad 1,-1, \quad 1, \quad 0, \quad 0, \quad 0, \cdots)\} \text {, }
\end{aligned}
$$




$$
\begin{aligned}
& S_{3}=\{(-1,-1,-1,-1, \quad 1, \quad 1, \quad 1, \quad 1, \quad 0, \quad 0, \quad 0, \cdots) \text {, } \\
& (-1,-1, \quad 1, \quad 1,-1,-1, \quad 1, \quad 1, \quad 0, \quad 0, \quad 0, \cdots) \text {, } \\
& (-1, \quad 1,-1, \quad 1,-1, \quad 1,-1,1, \quad 0,0,0, \cdots)\} \text {, }
\end{aligned}
$$

and so on. Then $\left|S_{n}\right|=2^{n} n$. Denote the sequences which make up $S_{n}$, taken in the order indicated, by $R_{1}^{n}, R_{2}^{n}, \cdots, R_{n}^{n}$, and denote the $m$ th term of $R_{k}^{n}$ by $R_{k}^{n}(m)$. Then

$$
\sum_{m=1}^{\infty}\left|\sum_{k \in \sigma} R_{k}^{n}(m)\right|=2^{n} \int_{0}^{1}\left|\sum_{k \in \sigma} r_{k}(t)\right| d t \leqq 2^{n} n^{1 / 2},
$$

as in Corollary 1 . We then have $\left|S_{n}\right|^{*} /\left|S_{n}\right| \rightarrow 0$, and the result follows as before.

\section{REFERENCES}

1. S. Banach, Théorie des opérations linéaires, Warsaw, 1932.

2. S. Kaczmarz and H. Steinhaus, Theorie der Orthogonalreihen, Warsaw and Lwów, 1935.

3. W. Orlicz, Über unbedingte Konvergenz in Funktionenräumen (II), Studia Mathematica vol. 4 (1933) pp. 41-47.

ACADIA UNIVERSITY 